\title{
The Usefulness of Super-Selective Computed Tomography Angiography (CTA) for Diagnosing and Localizing a Small Insulinoma
}

\author{
Akihiro Katayama ${ }^{1}$, Izumi Iseda ${ }^{1}$, Atsuhito Tone ${ }^{1}$, Yuichi Matsushita ${ }^{1}$, Kentaro Inoue ${ }^{1}$, \\ Keiko Tsukamoto $^{1}$, Haruhiro Yamashita ${ }^{2}$, Ichiro Yamadori ${ }^{3}$, Jun Wada ${ }^{4}$ and Kazuyuki Hida ${ }^{1}$
}

\begin{abstract}
Localization of insulinomas by preoperative imaging is critical for successful surgical resection. However, the visualization and localization of small insulinomas by recent imaging modalities still remains a challenge. Here, we report a 77-year-old woman with a small insulinoma successfully localized by performing arterial stimulation and venous sampling (ASVS), and subsequent super-selective CTA (SSCTA). It was not visualized by routine non-invasive imaging tests such as digital subtraction angiography (DSA). The small size (1.0 $\mathrm{cm}$ ) of the surgically removed tumor supports the usefulness of SSCTA for localizing very small insulinomas.
\end{abstract}

Key words: insulinoma, computed tomography angiography (CTA), imaging diagnosis, localization

(Inter Med 49: 1983-1986, 2010)

(DOI: 10.2169/internalmedicine.49.3739)

\section{Introduction}

Insulinomas are the most common functioning neoplasm of the pancreatic $\beta$ cells which provoke hyperinsulinemic hypoglycemia. Although they are associated with clinically specific symptoms, the preoperative diagnosis and localization is challenging because approximately $80-90 \%$ of the tumors are smaller than $2 \mathrm{~cm}$ in diameter $(1,2)$. Recent technical advances in imaging including contrast-enhanced ultrasonography (US), endoscopic US, multidetector CT, magnetic resonance imaging (MRI) with better gradients and coils, and DSA have improved considerably both in quality and sensitivity for the detection of insulinomas, however, we still confront difficulties in localizing small insulinomas (1-3). In this report, we present a case with a very small insulinoma, in which we successfully localized and surgically removed it by employing SSCTA.

\section{Case Report}

A 77-year-old woman was admitted to our hospital, since she had been suffered from repeated attacks of unconsciousness for 3 years. On admission, her height was $144 \mathrm{~cm}$, body weight $46 \mathrm{~kg}$ (BMI $22.1 \mathrm{~kg} / \mathrm{m}^{2}$ ) and waist circumstance $88 \mathrm{~cm}$. During past 3 years, she gained about $5 \mathrm{~kg}$. All laboratory data were within normal range, except for very low fasting plasma glucose level (FPG), $32 \mathrm{mg} / \mathrm{dL}$. The concurrent insulin level was not suppressed, $5.7 \mu \mathrm{U} / \mathrm{mL}$, under the condition of hypoglycemia. Despite hypoglycemia, glucagon, adrenaline and cortisol were within normal range, $124 \mathrm{pg} / \mathrm{mL}, 18 \mathrm{pg} / \mathrm{mL}$, and $15.5 \mu \mathrm{g} / \mathrm{dL}$, respectively. It suggested that the presence of hyporesponsiveness of counterregulatory hormones due to repeated hypoglycemic attacks. Although insulinoma was easily suspected from her symptoms and laboratory data, imaging techniques including US, contrast-enhanced helical CT and MRI did not show any ab-

\footnotetext{
${ }^{1}$ Department of Diabetology and Metabolism, National Hospital Organization, Okayama Medical Center, Okayama, ${ }^{2}$ Department of Gastroenterology, National Hospital Organization, Okayama Medical Center, Okayama, ${ }^{3}$ Department of Pathology, National Hospital Organization, Okayama Medical Center, Okayama and ${ }^{4}$ Department of Medicine and Clinical Science, Okayama University Graduate School of Medicine, Okayama
}

Received for publication April 1, 2010; Accepted for publication May 28, 2010

Correspondence to Dr. Kazuyuki Hida, kzhi@okayama3.hosp.go.jp 

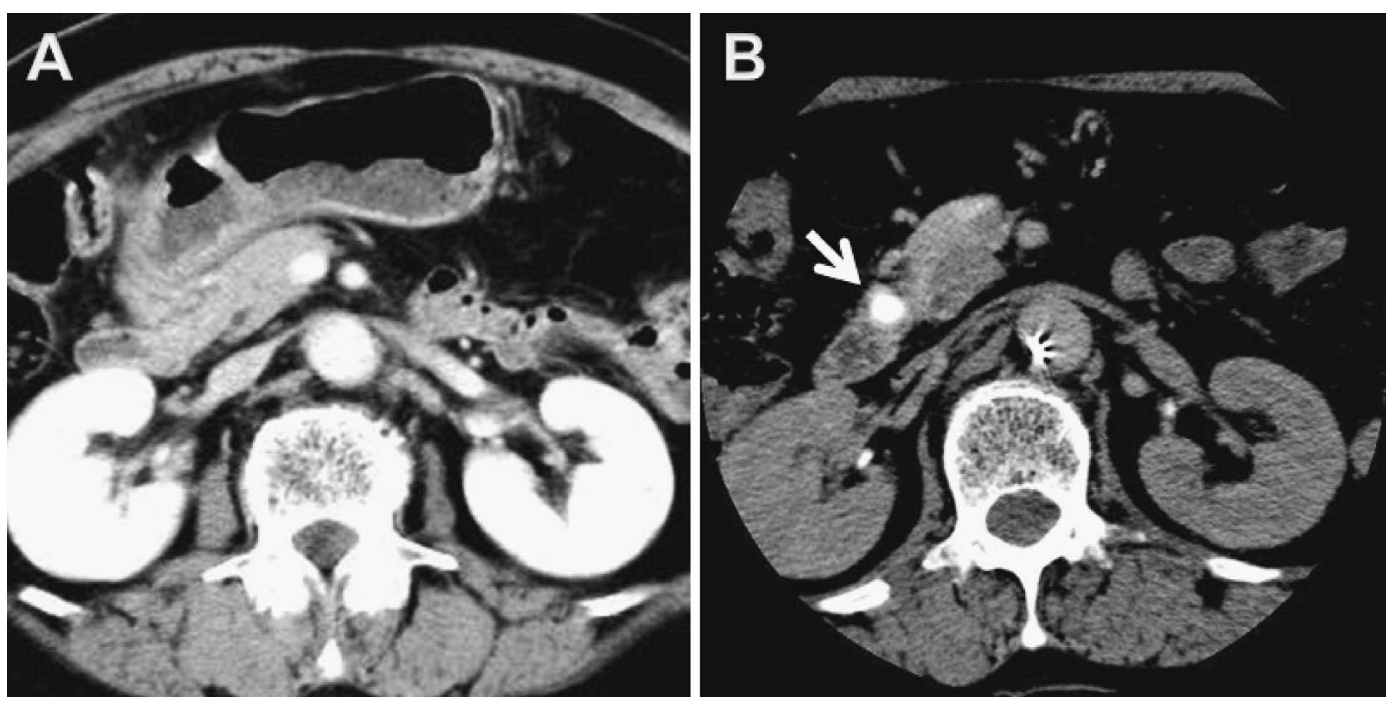

Figure 1. (A) Dynamic helical CT, which fails to show the tumor in the pancreas. (B) SSCTA demonstrating a well-enhanced round lesion in the pancreatic head (arrow).

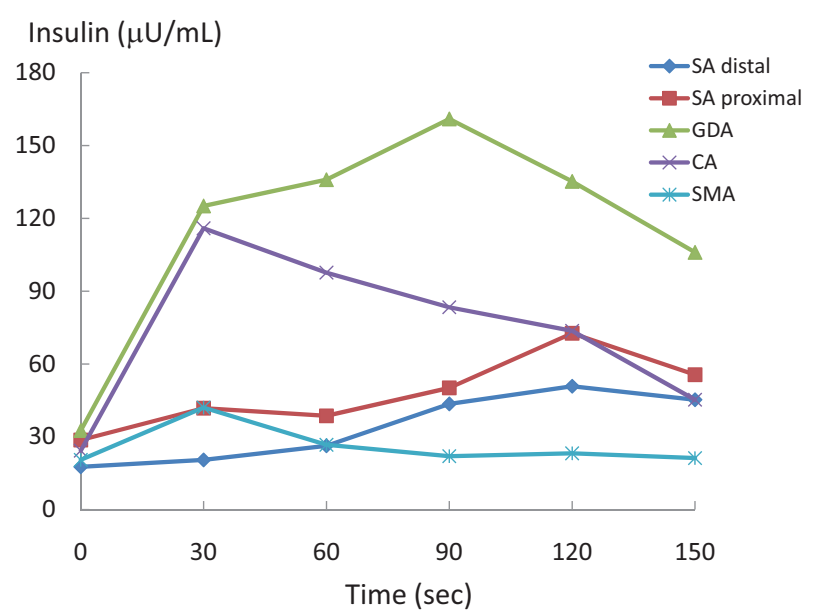

Figure 2. Graphs of insulin levels $(\mu \mathrm{U} / \mathrm{mL})$ after injection of calcium gluconate $(0.025 \mathrm{mEq} / \mathrm{kg})$. Four- to five-fold elevation of insulin is observed in GDA and CA. Abbreviations; SA: splenic artery, GDA: gastroduodenal artery, CA: celiac artery, SMA: superior mesenteric artery

normalities in her pancreas (Fig. 1A). DSA also failed to demonstrate abnormal tumor stains. After angiography, ASVS was subsequently performed and it showed a marked increase in insulin secretion after calcium stimulation into celiac artery (CA) and proximal gastroduodenal artery (GDA), indicating that the insulin secreting tumor located in the head of pancreas (Fig. 2). To further demonstrate the exact localization of the tumor, SSCTA was performed. Briefly, the catheter was super-selectively placed in the proximal GDA and $20 \mathrm{~mL}$ of contrast medium was injected through the catheter at an estimated $1.0 \mathrm{~mL} / \mathrm{sec}$ during sequential helical scanning of the pancreas. It showed a distinct round shape tumor measuring $1.0 \mathrm{~cm}$ in diameter (Fig. 1B). She was operated on and a $10 \times 9 \times 9 \mathrm{~mm}$ well bounded tumor was removed from the pancreas head by performing pancreatoduodenectomy (Fig. 3A). Acini of exocrine pancreas were seen surround the round tumor (Fig. 3B) and the strong immunoreactivities for insulin were visualized in the insulinoma cells (Fig. 3C). Two weeks after the operation, she was discharged with normal blood glucose profiles and the patient is still free of symptoms 4 years after the operation.

\section{Discussion}

The majority of insulinomas is single and benign $(1,4)$, which promises a hope for cure by successful surgical resection. Despite the availability of sophisticated modalities, localizing small insulinomas still remains a technical challenge (5). In a previous report, the positive rates for US, enhanced CT and angiography have been reported $48.9 \%, 80 \%$ and $69.3 \%$, respectively (6). ASVS has been developed as sensitive strategy for localization of insulinomas (6-9). It is invasive but powerful tool not only for rough localization of the tumors but for ascertaining the presence of functional tumor with high sensitivity (82\%) and specificity (91\%) (10). Preoperative examination of the further precise anatomical relationship between the tumor and the pancreatic duct is valuable and leads to a better surgical approach $(2,11,12)$. If insulinomas locate near the surface of the gland, simple enucleation may be performed to preserve the pancreatic parenchyma as much as possible. To minimize invasion, laparoscopic enucleations have been used for relatively small tumors $(<2 \mathrm{~cm}$ in diameter) and for tumors located on the surface of pancreas away from the pancreatic duct (13). In the present case, typical clinical symptoms and the result of ASVS indicating the tumor localization in the head of pancreas, prompted us to pinpoint its localization by performing SSCTA. It visualized well-defined $1.0 \mathrm{~cm}$ enhancing mass deep in the parenchyma of the pancreatic head. SSCTA possesses a clear advantage over conventional dynamic CT and 

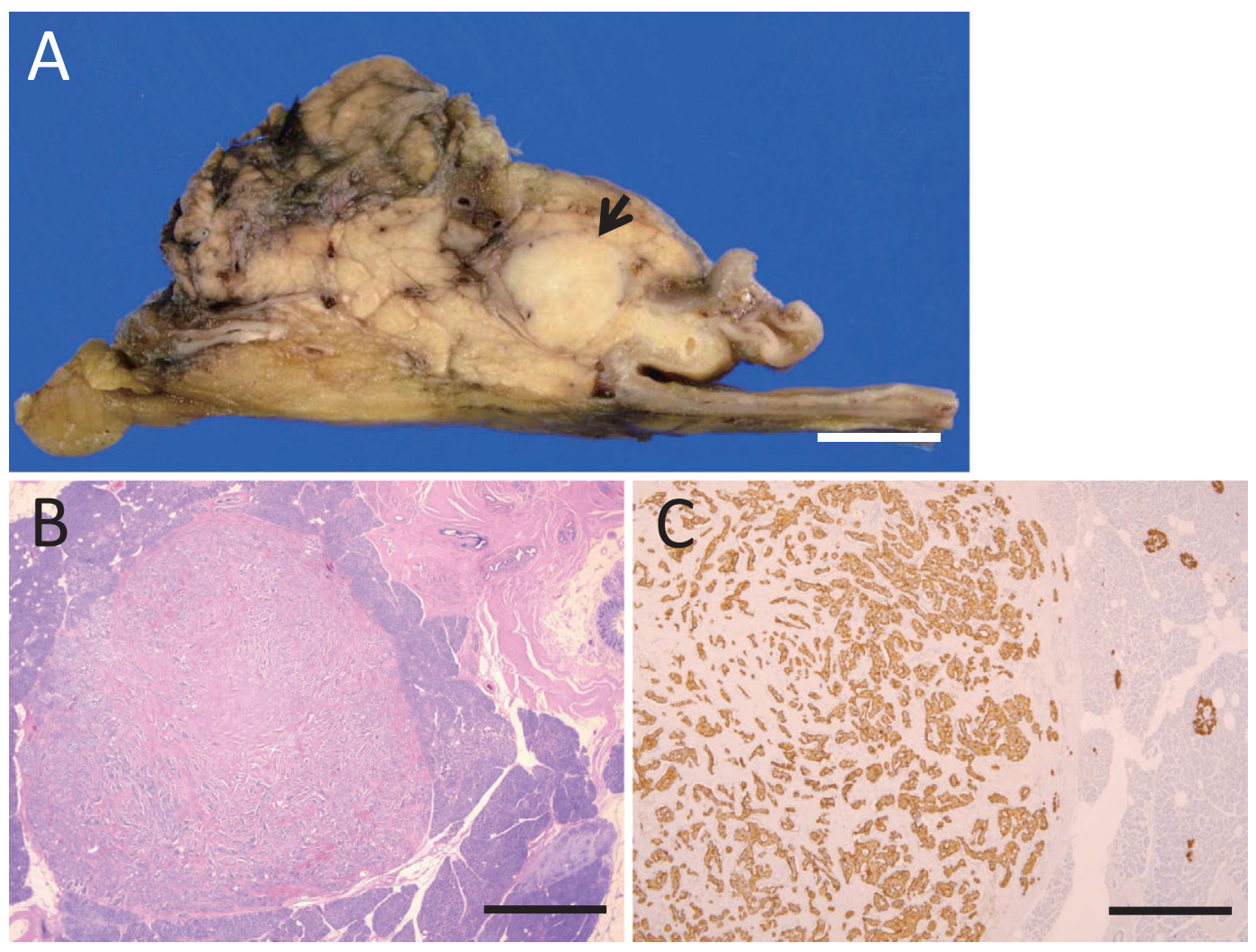

Figure 3. (A) Gross appearance of pancreas head. Well-bounded tumor, $10 \times 9 \times 9 \mathrm{~mm}$, is seen (arrow) (Bar=10 mm). (B) Insulinoma and surrounding normal acini of exocrine pancreas. Hematoxylin and Eosin staining. (C) Immunostaining for insulin. The strong immunoreactivities are seen. $($ Bar=2 mm)

it can provide greater enhancement of pancreatic tumors by the administration of contrast material directly into the proper artery. In the present case, super-selective injection of contrast material into the GDA is a key for success. An additional advantage in SSCTA is that it demonstrates the detailed relationship between the tumor and pancreatic parenchyma by $2 \mathrm{D}$ reconstructions such as horizontal, coronal and sagittal section. We suggest that SSCTA should be performed in ASVS-positive insulinoma in cases of failure in visualizing tumors by various imaging modalities. The major advantages of SSCTA over ASVS are its ability to reveal exact localization and detecting multiple tumors. The only disadvantage of SSCTA is that it requires an invasive and complicated procedure. Future studies are required to demonstrate the detection limit of size, sensitivity and specificity of SSCTA.

In conclusion, it is essential to visualize and localize insulinomas preoperatively to define the optimal surgical approach. We would like to postulate that SSCTA may be a high sensitive and invaluable imaging technique for visualizing small insulinomas when other sophisticated imaging approaches fail to detect.

\section{References}

1. Grant CS. Insulinoma. Best Pract Res Clin Gastroenterol 19: 783798, 2005.
2. Tucker ON, Crotty PL, Conlon KC. The management of insulinoma. Br J Surg 93: 264-275, 2006.

3. Chatziioannou A, Kehagias D, Mourikis D, et al. Imaging and localization of pancreatic insulinomas. Clin Imaging 25: 275-283, 2001.

4. van Heerden JA, Edis AJ, Service FJ. The surgical aspects of insulinomas. Ann Surg 189: 677-682, 1979.

5. Gouya H, Vignaux O, Augui J, et al. CT, endoscopic sonography, and a combined protocol for preoperative evaluation of pancreatic insulinomas. AJR Am J Roentgenol 181: 987-992, 2003.

6. Tsuzuki Y, Ishii H. Insulinoma-A statistical review of 358 cases of insulinoma reported from 1991 to 2000 in Japan. Nippon Rinsho 59: 121-131, 2001 (in Japanese).

7. Doppman JL, Miller DL, Chang R, Shawker TH, Gorden P, Norton JA. Insulinomas: localization with selective intraarterial injection of calcium. Radiology 178: 237-241, 1991.

8. Pereira PL, Roche AJ, Maier GW, et al. Insulinoma and islet cell hyperplasia: value of the calcium intraarterial stimulation test when findings of other preoperative studies are negative. Radiology 206: 703-709, 1998.

9. Morita S, Machida H, Kuwatsuru R, et al. Preoperative localization of pancreatic insulinoma by super selective arterial stimulation with venous sampling. Abdom Imaging 32: 126-128, 2007.

10. Lo CY, Lam KY, Kung AW, Lam KS, Tung PH, Fan ST. Pancreatic insulinomas. A 15-year experience. Arch Surg 132: 926-930, 1997.

11. Park BJ, Alexander HR, Libutti SK, et al. Operative management of islet-cell tumors arising in the head of the pancreas. Surgery 124: 1056-1061, 1998; discussion 1061-1062.

12. Abboud B, Boujaoude J. Occult sporadic insulinoma: localization 
Inter Med 49: 1983-1986, 2010 DOI: 10.2169/internalmedicine.49.3739

and surgical strategy. World J Gastroenterol 14: 657-665, 2008.

13. Tagaya N, Kasama K, Suzuki N, et al. Laparoscopic resection of the pancreas and review of the literature. Surg Endosc 17: 201206, 2003.

(C) 2010 The Japanese Society of Internal Medicine http://www.naika.or.jp/imindex.html 